\title{
Two-year treatment patterns and costs in glaucoma patients initiating treatment with prostaglandin analogs
}

This article was published in the following Dove Press journal:

Clinical Ophthalmology

28 September 2010

Number of times this article has been viewed

\section{Jordana K Schmier \\ Edmund C Lau ${ }^{2}$ \\ David W Covert ${ }^{3}$}

'Exponent, Alexandria, VI, USA; ${ }^{2}$ Exponent, Menlo Park, CA, USA; ${ }^{3}$ Alcon Research Ltd, Fort Worth, TX, USA
Correspondence: Jordana K Schmier 1800 Diagonal Road, Suite 500,

Alexandria, VA 22314, USA

$\mathrm{Tel}+\mid$ 57| $227724 \mid$

Fax + I 57I 2277299

Email jschmier@exponent.com
Objective: To determine treatment patterns and costs over a two-year period among new initiators of topical prostaglandin analogs in a managed care population by retrospective cohort analysis of an insurance claims database.

Methods: Patients who initiated therapy with a prostaglandin analog between September 2006 and March 2007 were identified. The use of monotherapy and adjunctive therapies were compared by index prostaglandin. Days to initiation of adjunctive therapy and rates of glaucoma surgical procedures were also calculated. Medical costs (antiglaucoma medications and ophthalmic visits) over the two-year period were estimated.

Results: The analysis identified 5018 patients with at least one prostaglandin analog prescription (bimatoprost, $\mathrm{n}=747$; latanoprost, $\mathrm{n}=1651$; benzalkonium chloride (BAK)-free travoprost, $\mathrm{n}=203)$. The majority $(51 \%-54 \%)$ had repeat prescriptions. Among those with repeat prescriptions, 52\% were female (not significant) and mean age was 64 years $(P<0.01)$. Rates of adjunctive therapy use varied across groups (bimatoprost $51 \%$, latanoprost $37 \%$, and BAK-free travoprost $35 \%, P<0.0001$ ). Median and mean days to initiation of adjunctive therapy were 83 and 140 for bimatoprost, 101 and 181 for latanoprost, and 113 and 221 for BAK-free travoprost. Two-year medical costs were \$3147, \$2843, and \$2557 for patients initiating treatment with bimatoprost, latanoprost, and BAK-free travoprost, respectively. Use of glaucoma surgical procedures across the treatment groups was similar over the two-year period.

Conclusions: Over a two-year period, the rate and time to initiation of adjunctive therapy use, as well as medical costs, varied between index prostaglandins. However, the rate of glaucoma surgical interventions did not vary significantly across index medications.

Keywords: costs and cost analysis, drug therapy, combination, glaucoma, prostaglandin analogs

\section{Introduction}

Patient adherence to glaucoma treatment regimens is essential to maintain maximum intraocular pressure (IOP) reduction and to delay disease progression in patients with primary open-angle glaucoma. ${ }^{1}$ However, adherence with glaucoma treatment is a known problem, with multiple studies suggesting that more than half of glaucoma patients who initiate treatment fail to refill prescriptions. ${ }^{2-6}$ Reasons for nonadherence can vary, ranging from patient characteristics, such as race, travel, or financial challenges, to poor patient-doctor communication, limited understanding of the risks of nonadherence, or not being willing to discuss adverse events with the physician. ${ }^{7}$ Some of these reasons are common across medications and medication classes, but adverse events is one area in which differences between medications may have an impact on patient adherence and treatment efficacy. For example, recent estimates suggest that ocular surface disease (OSD) is experienced by more than half of all glaucoma 
patients. ${ }^{8}$ Limiting the use in IOP-lowering medications of the preservative benzalkonium chloride (BAK), which is associated with increased rates of $\mathrm{OSD}^{8,9}$ may minimize these rates.

Minimizing preservatives such as BAK can be accomplished in a number of ways and can have important longterm eye health benefits. Using a BAK-free product and limiting exposure by prescribing monotherapy instead of polypharmacy are ideal ways to do this, but monotherapy may not be sufficient to lower IOP adequately and adjunctive therapies are required. The introduction of adjunctive therapies multiplies negative influences on adherence (ie, complexity of regimen, likelihood of adverse events) $)^{10,11}$ and increases exposure to BAK which has been linked to decreased success with filtration surgery. ${ }^{12}$ Therefore, the simplest treatment regimen, with lowest exposure to BAK and the least need for adjunctive therapy, is optimal. Not only does increased medication complexity decrease adherence, ${ }^{10,13}$ but adverse events such as OSD associated with BAK can affect adherence. ${ }^{7}$

In theory, these recommendations seem reasonable, but only a real-world comparison of treatments can address the issue adequately. Several studies with one-year follow-up of patients treated with a BAK-free prostaglandin analog (BAK-free travoprost) compared with those treated with alternative prostaglandin analogs (bimatoprost and latanoprost) have been published. ${ }^{4-6}$ Each study used the same inclusion criteria and identified patients with a prescription for a topical prostaglandin analog during a six-month period and who had no glaucoma therapy claims in the six months prior and had at least 12 months of data available after the initial prostaglandin analog claim. These studies have consistently found that during the first year of treatment, a BAK-free prostaglandin analog was associated with lower rates of adjunctive therapy, a longer time until initiating adjunctive therapy, and lower first-year costs.

While these studies present evidence that BAK-free travoprost provides treatment advantages within a single year of follow-up, it was useful to extend this analysis to include two years and to examine glaucoma surgical procedures as a possible confounder of previous study findings, ie, whether surgical procedures were performed at different rates across groups and thus affected the need for further pharmacotherapy. The goals of this study are similar to the previous analyses, ie, to evaluate the treatment patterns and costs of patients newly initiating therapy with prostaglandin analogs as first-line therapy. However, a different health care database was used, which included glaucoma surgical procedural data. Thus, this study includes an analysis of these findings, along with an evaluation of pharmaceutical treatment data over a two-year period.

\section{Methods}

The patients described in the retrospective cohort analysis were receiving prescription benefits and were included in the Integrated HealthCare Information Services National Managed Care Benchmark Database, a medical claims database covering managed care plans that serve more than 30 million members across the US. All data were deidentified in accordance with Protected Health Information standards under the Health Information Portability and Accountability Act, so that no individually identifiable information was included in the study database. Therefore, review by an institutional review board was not required.

The study cohort included patients who first initiated therapy with one of three prostaglandin analog products (bimatoprost, latanoprost, or BAK-free travoprost) between September 1, 2006 and March 30, 2007. To qualify, patients had to have at least one claim for primary open-angle glaucoma (International Classification of Diseases, Ninth Revision, Clinical Modification [ICD-9-CM] code 365.11) during the observation period and six months of prior claims data in which there were no glaucoma therapy claims of any class. The index date was defined as the first date on which a prostaglandin analog was filled. Glaucoma therapy claims were defined by National Drug Codes. The study population was further defined by requiring patients to have at least 24 months of uninterrupted use of the index prostaglandin analog following the index date (initial prescription fill). This was operationalized as not having prescriptions for any other prostaglandin analogs during the follow-up period and having at least one prescription for the index prostaglandin analog during months 19-24 of the follow-up period. Patients were required to be continuously enrolled, as per the eligibility database, during the 30-month observation period (six months prior to the first prostaglandin claim and the 24 months of follow-up). Patients who did not meet these requirements (sufficient prior claims data, uninterrupted use, and repeat use of the index prostaglandin) were excluded from the study database. We considered that patients added an adjunctive medication, defined by National Drug Code, if there was a sequential and subsequent refill of an adjunctive agent in the presence of continued refills for the index agent. Adjunctive medications were described by type (adrenergics, beta-blockers, carbonic anhydrase inhibitors, miotics, prostaglandin analogs, fixed combinations, others). 
The analysis identified patients by the prostaglandin prescription they first filled (ie, the "index medication") during the study period, with one exception. Patients who initiated treatment with the original formulation of travoprost but then switched to the BAK-free formulation after a single prescription for the original formulation were included in the BAK-free cohort. Participants were characterized in terms of available demographic characteristics (age and gender). Gender was compared using a Chi-square test and age was compared using analysis of variance (ANOVA). The use of monotherapy and adjunctive therapies was compared. Median and mean number of days to initiation of adjunctive therapy were calculated for each cohort, with the distribution of number of days examined to determine which measure to use in the model. The median numbers of days until patients added adjunctive therapies were compared using a Wilcoxon rank sum approach, and mean times were compared using ANOVA. The rate of selected surgical procedures for glaucoma, as specified in the published literature, ${ }^{14,15}$ was calculated over the observation period, with rates compared across patient cohorts, controlling for age and gender. Statistical comparisons were conducted in SAS v9.2 (SAS Institute, Inc., Cary, NC).

The American Academy of Ophthalmology Preferred Practice Patterns for glaucoma suggest that follow-up care should be based on achievement of target IOP and the amount of disease progression, ${ }^{16}$ neither of which was available in the prescription database used for this study. Therefore, the base case analysis uses resource rates from a survey-based study ${ }^{17}$ while sensitivity analyses explore visits, as recommended by other studies and guidelines. ${ }^{16,18}$ Because all patients in the model are assumed to undergo the same procedures and tests at their visits regardless of the prostaglandin analog they receive, the relatively small differences across studies in terms of the components of each visit have minimal impact on the study findings.

At the initial visit, patients are assumed to have a level 4 (comprehensive) evaluation (Current Procedural Terminology, 92004). Follow-up visits are assumed to be level 2 (intermediate) visits (Current Procedural Terminology 92012), with three follow-up visits during the first year (likely but not necessarily at 30 and 90 days following initiation of the index therapy and at 12 months, and then an additional two visits in the following year). Two additional follow-up visits are assumed to be associated with the initiation and monitoring of adjunctive medication among patients for whom it is prescribed. Thus, patients who stay on monotherapy are assumed to have one comprehensive visit and five intermediate visits, while those who require adjunctive therapy had one comprehensive visit and seven intermediate visits. Table 1 presents the procedures and diagnostic tests that comprised each visit, as well as the costs used for each.

We used average wholesale price (AWP) for 2009 as the basis of prescription costs. ${ }^{19}$ The range of published AWPs for the prostaglandin analogs was fairly narrow ( $\$ 80.53$ to \$82.67). ${ }^{19}$ For the prostaglandin analogs, the midpoint cost for the agents is used in the model because AWP is the best publicly available estimate for the analysis. Further, the number of prescriptions for prostaglandin analogs was expressed as $2.5 \mathrm{~mL}$ size bottle equivalents, because this is the most common size found in the claims database and represents approximately a 30-45 day supply when used as per label in both eyes. For example, a $5 \mathrm{~mL}$ bottle was treated as two $2.5 \mathrm{~mL}$ bottles. The AWPs for adjunctive therapies were also reviewed in the Red Book. ${ }^{19}$ For the base case of the model, branded products were used, but sensitivity analyses explored lower costs. Days of use per bottle are based on data from previously published studies. ${ }^{4,5}$ Number of bottles required is rounded to the nearest tenth. Medical charges are estimated using the 75th percentile of physicians' fees from a published benchmark. ${ }^{20}$ All the costs used in the model are presented in Table 2.

\section{Results}

A total of 5018 patients were identified who had six months of glaucoma-free eligibility and 24 months of continuous eligibility after an initial prescription for bimatoprost, latanoprost, or BAK-free travoprost (Figure 1). Of the 1382 bimatoprost patients, 747 had repeat prescriptions, and of the 3247 latanoprost patients, 1651 had repeat prescriptions. Among the 389 patients who initiated with BAK-free travoprost, 203 had repeat prescriptions (ie, no other prostaglandins during the follow-up period plus at least one repeat of the index prescription in months 19-24). Fifty-six of these 203 had an initial prescription with the original formulation of travoprost and were then transitioned to the BAK-free formulation. Persistence patterns differed only slightly across groups, with $54 \%$ of bimatoprost patients remaining on the

Table I Demographic characteristics

\begin{tabular}{llll}
\hline Characteristic & $\begin{array}{l}\text { BAK-free } \\
\text { travoprost }\end{array}$ & Bimatoprost & Latanoprost \\
\hline $\mathrm{N}$ & 203 & 747 & $165 \mathrm{I}$ \\
Age, mean \pm SD* & $62.1 \pm 10.7$ & $65.1 \pm 10.7$ & $64.1 \pm 11.5$ \\
Gender, \% female & $50.7 \%$ & $49.4 \%$ & $53.4 \%$ \\
\hline
\end{tabular}

Note: $* P=0.0096$ across treatment groups.

Abbreviations: BAK, benzalkonium chloride; SD, standard deviation. 
Table 2 Unit costs

\begin{tabular}{|c|c|c|}
\hline Resource & Description/Size & Cost* \\
\hline Initial visit & $\begin{array}{l}\text { CPT } 92004 \text { (comprehensive, } \\
\text { new patient) plus weighted } \\
\text { costs for visual field examination } \\
\text { (CPT 92082), gonioscopy } \\
\text { (CPT 92020), evaluation of optic } \\
\text { disc (CPT 92135), optic nerve } \\
\text { head photograph (CPT 92235), } \\
\text { and fundus evaluation (CPT 92250) } \\
\text { per Fremont et al }{ }^{17}\end{array}$ & $\$ 582.6 I^{20}$ \\
\hline Follow-up visit & $\begin{array}{l}\text { CPT } 92012 \text { (intermediate, } \\
\text { established patient) and visual } \\
\text { acuity examination (CPT 99/73) per } \\
\text { Fremont et al }{ }^{17}\end{array}$ & $\$ 117.68^{20}$ \\
\hline Prostaglandin analog & $2.5 \mathrm{~mL}$ & $\$ 81.60^{19}$ \\
\hline $\begin{array}{l}\text { Fixed-combination } \\
\text { dorzolamide } 2.0 \% \text { - } \\
\text { timolol } 0.5 \%\end{array}$ & $5 \mathrm{~mL}$ & $\$ 130.80^{19}$ \\
\hline Brimonidine $0.1 \%$ & $5 \mathrm{~mL}$ & $\$ 62.80^{19}$ \\
\hline Brinzolamide $1.0 \%$ & $10 \mathrm{~mL}$ & $\$ 91.38^{19}$ \\
\hline Timolol $0.25 \%$ & $5 \mathrm{~mL}$ & $\$ 17.93^{19}$ \\
\hline Other & $5 \mathrm{~mL}$ & $\begin{array}{l}\$ 75.73 \\
\text { (assumption) }\end{array}$ \\
\hline
\end{tabular}

Note: *Costs are expressed in 2009 US\$.

index therapy, while $51 \%$ of latanoprost patients and $52 \%$ of BAK-free travoprost patients remained on the index therapy. Figure 1 details the patient population persistence patterns.

Demographic characteristics by index medication are presented in Table 1. Average age across all groups was 64 years,

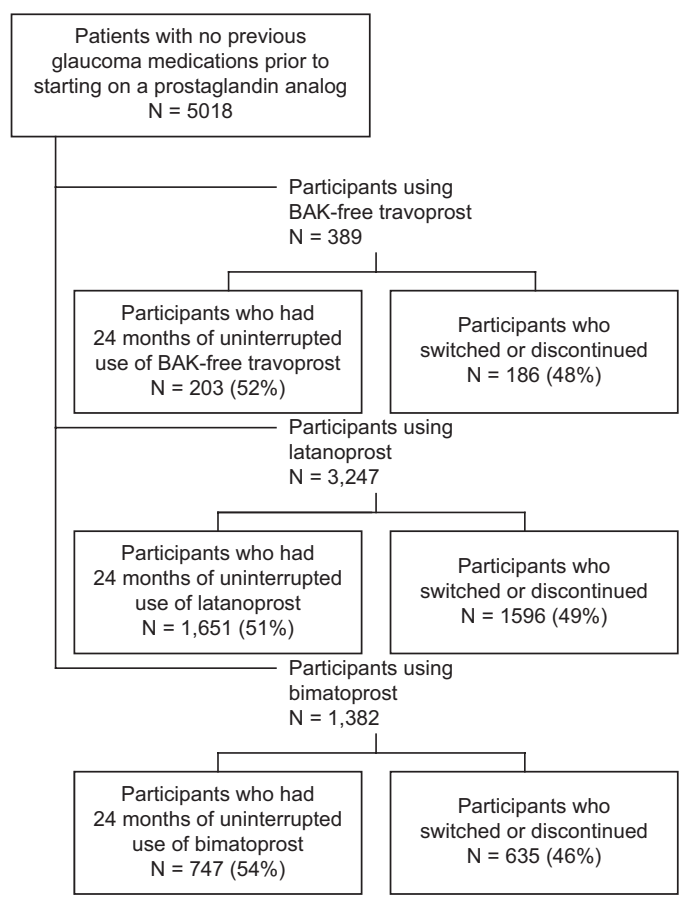

Figure I Patient selection. and $52 \%$ of the patients were female. Gender was similar across groups, although there were significant differences in age across groups (range was $62-65$ years, $P<0.01$ ).

In the bimatoprost, latanoprost, and BAK-free travoprost treatment groups, $51.3 \%, 37.3 \%$, and $35.0 \%$, respectively, initiated adjunctive therapy during the course of the year at significantly different proportions $(P<0.0001$, see Table 3 ). Patients on BAK-free travoprost were able to continue without adjunctive therapy longer than patients treated with other prostaglandins. The median number of days until the patients added adjunctive therapies was 83 for bimatoprost, 101 for latanoprost, and 113 for patients for BAK-free travoprost. The mean number of days to initiating adjunctive therapy were 221 in the BAK-free travoprost group, 140 for patients receiving bimatoprost, and 181 for patients receiving latanoprost $(P<0.0001)$.

The types of adjunctive therapies used also appeared to differ across index therapies, although comparisons are difficult to interpret because of the use of fixed combination therapies. For example, among patients treated with BAK-free travoprost and latanoprost, beta-blockers were the most commonly used adjunctive medication, while for the bimatoprost-treated patients, a fixed combination therapy of beta-blocker and carbonic anhydrase inhibitor was most common. However, across therapies, the rate of use of any beta-blocker (ie, alone or as part of a combination therapy) was fairly consistent, ranging from $60 \%$ to $64 \%$, and the rate of use of any carbonic anhydrase inhibitor was only slightly more variable $(35 \%-42 \%)$. Since the "other adjunctive therapy" category includes other fixed combination therapies, such as a combination of an $\alpha_{2}$-adrenegeric receptor agonist and beta-blocker, or different doses of medications, comparisons are not useful.

Medical costs, ie, ophthalmic visits and procedures (excluding surgical procedures), prostaglandin analogs, and adjunctive therapies, were estimated at $\$ 2557$ for patients initiating with BAK-free travoprost, $\$ 3147$ for patients initiating with bimatoprost, and $\$ 2843$ for patients initiating with latanoprost. Sensitivity analyses that explored clinical and cost parameters did not change the direction or magnitude of findings substantially. Surgical procedures were not included in the costs because of the low frequency and the similarity in rates across index prostaglandins.

Table 4 presents findings on the rate of surgical procedures for glaucoma among the study population. There were too few patients who received BAK-free travoprost to compare rates for certain procedures, but rates for most procedures were similar across treatment groups. 
Table 3 Treatment patterns and costs during observation period

\begin{tabular}{|c|c|c|c|}
\hline Treatment pattern & BAK-free travoprost & Bimatoprost & Latanoprost \\
\hline Number remaining on index therapy (n) & 203 & 747 & $165 \mid$ \\
\hline Remained on monotherapy (\%)** & $65.0 \%$ & $48.7 \%$ & $62.8 \%$ \\
\hline Required adjunctive therapy (\%)** & $35.0 \%$ & $51.3 \%$ & $37.3 \%$ \\
\hline Median time to adding adjunctive therapy (days) & 113 & 83 & 101 \\
\hline Mean time to adding adjunctive therapy (days); & $221 \pm 223$ & $140 \pm 156$ & $181 \pm 187$ \\
\hline mean $\pm S D$ (interquartile range)** & $(56-397)$ & $(34-175)$ & $(4 I-263)$ \\
\hline \multicolumn{4}{|l|}{ Type of adjunctive therapy } \\
\hline$\alpha_{2}$-adrenergic receptor agonist (\%) & $19.7 \%$ & $26.1 \%$ & $24.6 \%$ \\
\hline Beta-blocker (\%) & $35.2 \%$ & $24.5 \%$ & $34.1 \%$ \\
\hline Carbonic anhydrase inhibitor (\%) & $7.0 \%$ & $7.0 \%$ & $5.4 \%$ \\
\hline Fixed-combination beta-blocker- carbonic anhydrase-inhibitor (\%) & $28.2 \%$ & $35.2 \%$ & $29.4 \%$ \\
\hline Other adjunctive therapy (\%) & $9.9 \%$ & $7.0 \%$ & $6.5 \%$ \\
\hline Number of prescriptions of index prostaglandin & $10.7 \pm 6.2$ & $13.5 \pm 7.6$ & $13.6 \pm 9.5$ \\
\hline Total costs, 24 months & $\$ 2556.87$ & $\$ 3147.40$ & $\$ 2842.60$ \\
\hline
\end{tabular}

Note: $* * P<0.0001$ across treatment groups.

Abbreviations: BAK, benzalkonium chloride; SD, standard deviation.

\section{Discussion}

This analysis adds to findings from previous studies that explored the one-year treatment patterns of patients initiating on prostaglandin analogs $\mathrm{s}^{4,5}$ by providing a more comprehensive and longer follow-up analysis. The extended follow-up period (24 versus 12 months) and availability of procedural data provides information on long-term patterns of care for patients with glaucoma not available in previous published studies. While previous studies found rates of adjunctive therapy use to range from $12 \%$ (BAK-free travoprost ${ }^{6}$ ) to $23 \%$ (bimatoprost $^{6}$ ) over one year, we found that this increased to a range of $35 \%$ (BAK-free travoprost) to $51 \%$ (bimatoprost) of patients requiring adjunctive therapy over two years. Interestingly, the median time to adding adjunctive therapy was in the same range for BAK-free travoprost (116 days in this analysis versus 109-159 days in previous analyses) while the median times to initiating adjunctive therapy with bimatoprost or latanoprost were longer in this analysis than in previous analyses (for bimatoprost 83 days compared with 53-73 days in previous studies; for latanoprost 101 days compared with $63-74$ days in previous studies). ${ }^{4,5} \mathrm{~A}$ full electronic health record might provide more information explaining these differences, and a meta-analysis of previous studies suggests that there may be significant differences in efficacy across these medications. ${ }^{21}$

Consistent with previous studies, the average cost per patient over the observation period was lowest in the cohort with the longest duration of monotherapy. Since the difference in monotherapy rates was more than $15 \%$ (48.7\% for bimatoprost and $65.0 \%$ for BAK-free travoprost), the additional follow-up visits for patients who switched therapies also had an impact on the cost differences. In fact, when the rates

Table 4 Rate of surgical procedures during observation period (number per patient)

\begin{tabular}{|c|c|c|c|c|}
\hline Procedure & CPT & BAK-free travoprost & Bimatoprost & Latanoprost \\
\hline Laser trabeculoplasty' & 68566 & 0.000 & 0.000 & 0.000 \\
\hline Trabeculectomy, no previous surgery ${ }^{2}$ & 66170 & 0.005 & 0.024 & 0.010 \\
\hline Trabeculectomy, previous surgery or trauma ${ }^{2}$ & 66172 & 0.015 & 0.007 & 0.004 \\
\hline Aqueous shunt to reservoir ${ }^{2}$ & 66180 & 0.034 & 0.013 & 0.010 \\
\hline Revision of aqueous shunt ${ }^{3}$ & 66185 & 0.000 & 0.003 & 0.002 \\
\hline Iridectomy, peripheral, for glaucoma ${ }^{3}$ & 66625 & 0.000 & 0.000 & 0.000 \\
\hline Iridectomy, sector, for glaucoma' & 66630 & 0.000 & 0.000 & 0.000 \\
\hline Cyclophotocoagulation, transscleral ${ }^{2}$ & $66710 *$ & 0.005 & 0.003 & 0.006 \\
\hline Cyclophotocoagulation, endoscopic ${ }^{4}$ & 66711 & 0.000 & 0.007 & 0.001 \\
\hline Laser iridotomy ${ }^{2}$ & 66761 & 0.015 & 0.033 & 0.036 \\
\hline Laser iridoplasty' & 66762 & 0.000 & 0.003 & 0.005 \\
\hline Total & & 0.074 & 0.093 & 0.078 \\
\hline
\end{tabular}

Notes: *In 2006, this included 667II; 'none performed; ${ }^{2}$ not significantly different across treatment groups; ${ }^{3}$ no significant difference between bimatoprost and latanoprost; comparison with BAK-free travoprost could not be performed due to sample size; ${ }^{4}$ rate significantly lower for latanoprost than bimatoprost, BAK-free travoprost rate similar to bimatoprost.

Abbreviations: BAK, benzalkonium chloride, CPT, Current Procedural Terminology. 
of monotherapy were equalized across cohorts in sensitivity analyses, the magnitude of the difference in total costs was halved.

Previous analyses for which only prescription claims data were available left open the question about whether low persistence was associated with the use of glaucoma surgical interventions. That is, were patients able to discontinue therapies because of a successful glaucoma surgical intervention? While these data do not show the success rates of interventions, the analysis demonstrated very low use of glaucoma surgical interventions across index medications in the first two years after initiating treatment. Therefore, for those patients who are new to glaucoma therapy, surgical interventions would not be the reason for the high rate of patients stopping all glaucoma medications.

As with any claims database analysis, there are certain limitations that should be acknowledged. The use of a large claims database offers an important strength in terms of sample size but shortcomings remain. For example, the patient population in any given database may not match the general population of patients with the condition in question, although in this analysis, the age and gender of the population reflect that of the general glaucoma population. ${ }^{22}$ Other characteristics, such as race, educational status, or self-reported health status, among others, are unknown. Along those lines, it is important to note that patients in the study database were not randomized to treatment. Further, various clinical or personal characteristics that may be associated with treatment assignment cannot be known from the database. Some clinical characteristics can be examined, eg, there is some evidence that glaucoma patients taking antihypertensive drugs may have different adjunctive therapy rates ${ }^{23}$ but we did not undertake such an analysis. The population of prescribers may not accurately reflect national patterns, which could affect generalizability. Similarly, the treatment selection process may vary based on region, past success with the treatment, and many other factors. ${ }^{24,25}$ Most claims databases have little or no data on the characteristics of prescribers but, because the data were from a large managed care organization, it is likely that there is a wide distribution of prescribers and practices. By their nature, claims databases only provide information on people with some type of insurance coverage, and those with coverage may be more likely to seek out and adhere to treatment. Finally, claims databases may contain coding biases or errors, although there is no reason to believe that these errors would be different across index treatments. In addition, the claims in this database were reviewed and adjudicated before the database was prepared for this analysis.
The methodology for patient selection in this study is also subject to certain limitations. The study attempted to identify only patients who were new to therapy by requiring six months of glaucoma-free claims prior to the first prostaglandin analog prescription. However, there is no way to account for product samples, which one study identified as being received by $20 \%$ of patients, ${ }^{3}$ and which could translate into patients who were already taking prostaglandin analogs before our study identified them. Also, based on this study methodology, patients with poor adherence could have been included, because patients in the cohort analysis were required to have a minimum of two glaucoma prescriptions filled during the 24-month follow-up period, but no attempt was made to assess medication possession ratio.

\section{Conclusion}

This study found that over two years, the rate of adjunctive therapy use was lower and time to initiating adjunctive therapy was longer with a BAK-free prostaglandin analog compared with other alternatives. The reasons for this advantage are unknown. It cannot be ruled out that differences in physicians' prescribing patterns affects these findings. This difference in treatment patterns was not associated with glaucoma surgical interventions, which were at a low rate of use and similar across patient cohorts. Different rates of use of adjunctive therapy led to a substantial difference in costs over a two-year period. A full medical record review could help explore the reasons for low persistence rates and the advantages to minimizing BAK in the treatment of patients with primary open-angle glaucoma.

\section{Disclosure}

Funding for this research was provided by Alcon Research, Ltd. One author (DC) is an employee of Alcon; the others (JS, EL) work for Exponent, which received a grant from Alcon to conduct this research.

\section{References}

1. Konstas AG, Maskaleris G, Gratsonidis S, Sardelli C. Compliance and viewpoint of glaucoma patients in Greece. Eye (Lond). 2000;14(Pt 5): $752-756$.

2. Yeaw J, Benner JS, Walt JG, Sian S, Smith DB. Comparing adherence and persistence across 6 chronic medication classes. J Manag Care Pharm. 2009;15(9):728-740.

3. Friedman DS, Quigley HA, Gelb L, et al. Using pharmacy claims data to study adherence to glaucoma medications: Methodology and findings of the Glaucoma Adherence and Persistency Study (GAPS). Invest Ophthalmol Vis Sci. 2007;48(11):5052-5057.

4. Schmier JK, Covert DW, Robin AL. First-year treatment patterns among new initiators of topical prostaglandin analogs. Curr Med Res Opin. 2009;25(4):851-858.

5. Schmier JK, Covert DW, Robin AL. First-year treatment costs among new initiators of topical prostaglandin analogs. Clin Ophthalmol. 2009;3: $637-644$. 
6. Schmier JK, Covert DW. First-year treatment costs among new initiators of topical prostaglandin analogs: Pooled results. Clin Ophthalmol. 2010;4:437-445.

7. Friedman DS, Hahn SR, Gelb L, et al. Doctor-patient communication, health-related beliefs, and adherence in glaucoma results from the Glaucoma Adherence and Persistency Study. Ophthalmology. 2008; 115(8):1320-1327.

8. Leung EW, Medeiros FA, Weinreb RN. Prevalence of ocular surface disease in glaucoma patients. J Glaucoma. 2008;17(5): 350-355.

9. Fechtner RD, Godfrey DG, Budenz D, Stewart JA, Stewart WC, Jasek MC. Prevalence of ocular surface complaints in glaucoma patients using topical intraocular pressure-lowering medications. Cornea. 2010; 29(6):618-621.

10. Robin AL, Novack GD, Covert DW, Crockett RS, Marcic TS. Adherence in glaucoma: Objective measurements of once-daily and adjunctive medication use. Am J Ophthalmol. 2007;144(4):533-540.

11. Tsai JC. A comprehensive perspective on patient adherence to topical glaucoma therapy. Ophthalmology. 2009;116 Suppl 11 :S30-S36.

12. Broadway DC, Grierson I, O’Brien C, Hitchings RA. Adverse effects of topical antiglaucoma medication. II. The outcome of filtration surgery. Arch Ophthalmol. 1994;112(11):1446-1154.

13. Robin AL, Covert D. Does adjunctive glaucoma therapy affect adherence to the initial primary therapy? Ophthalmology. 2005;112(5): 863-868.

14. Ramulu PY, Corcoran KJ, Corcoran SL, Robin AL. Utilization of various glaucoma surgeries and procedures in Medicare beneficiaries from 1995 to 2004. Ophthalmology. 2007;114(12):2265-2270.

15. Schmier JK, Covert DW, Lau EC, Robin AL. Trends in annual medicare expenditures for glaucoma surgical procedures from 1997 to 2006. Arch Ophthalmol. 2009;127(7):900-905.
16. American Academy of Ophthalmology. Preferred Practice Pattern. Primary Open-Angle Glaucoma. Limited Revision. San Francisco, CA: American Academy of Ophthalmology; 2003.

17. Fremont AM, Lee PP, Mangione CM, et al. Patterns of care for open-angle glaucoma in managed care. Arch Ophthalmol. 2003;121(6):777-783.

18. Quigley HA, Friedman DS, Hahn SR. Evaluation of practice patterns for the care of open-angle glaucoma compared with claims data: The Glaucoma Adherence and Persistency Study. Ophthalmology. 2007; 114(9):1599-1606.

19. Thomson Healthcare. Red Book Drug Topics. Montvale, NJ: Thomson Healthcare; 2009.

20. Physicians' Fee Reference: Comprehensive Fee Report: Milwaukee, WI: Yale Wasserman DMD. Medical Publishers; 2009.

21. Denis P, Lafuma A, Khoshnood B, Mimaud V, Berdeaux G. A metaanalysis of topical prostaglandin analogues intra-ocular pressure lowering in glaucoma therapy. Curr Med Res Opin. 2007;23(3):601-608.

22. Friedman DS, Wolfs RC, O'Colmain BJ, et al. Prevalence of openangle glaucoma among adults in the United States. Arch Ophthalmol. 2004;122(4):532-538

23. Iskedjian M, Walker JH, Desjardins O, et al. Effect of selected antihypertensives, antidiabetics, statins and diuretics on adjunctive medical treatment of glaucoma: A population based study. Curr Med Res Opin. 2009;25(8):1879-1888.

24. Nutescu EA, Park HY, Walton SM, et al. Factors that influence prescribing within a therapeutic drug class. J Eval Clin Pract. 2005; 11(4):357-365.

25. Schumock GT, Walton SM, Park HY, et al. Factors that influence prescribing decisions. Ann Pharmacother. 2004;38(4):557-562.
Clinical Ophthalmology

\section{Publish your work in this journal}

Clinical Ophthalmology is an international, peer-reviewed journal covering all subspecialties within ophthalmology. Key topics include: Optometry; Visual science; Pharmacology and drug therapy in eye diseases; Basic Sciences; Primary and Secondary eye care; Patient Safety and Quality of Care Improvements. This journal is indexed on

Submit your manuscript here: http://www.dovepress.com/clinical-ophthalmology-journal

\section{Dovepress}

PubMed Central and CAS, and is the official journal of The Society of Clinical Ophthalmology (SCO). The manuscript management system is completely online and includes a very quick and fair peer-review system, which is all easy to use. Visit http://www.dovepress.com/ testimonials.php to read real quotes from published authors. 